\title{
Performance Comparation of TR and FDE in the MB - IR - UWB System Based on MIMO
}

\author{
Yu Xin, Qi Lin, Dou Zheng and Lin Yun \\ School of Information and Communication Engineering, Harbin Engineering \\ University, Harbin, Heilongjiang, 150001, China \\ yuxin123@hrbeu.edu.cn,qilin@hrbeu.edu.cn,douzheng@hrbeu.edu.cn, \\ linyun.phd@gmail.com
}

\begin{abstract}
In order to improve the channel capacity of the multiband impulse radio ultra-wideband (MB-IR-UWB) system, the multiple input and multiple output (MIMO) technology is introduced to MB-IR-UWB system, and we get a new MB-IR-UWB system based on MIMO. But the introduction of MIMO technology brings multi-stream interference (MSI) and same frequency interference (SFI). Meanwhile, there may be inter-symbol interference (ISI) over the multipath channel proposed by IEEE 802.15.3a. To eliminate these interferences, it is necessary to add an equalization technique which is a technique used for increasing the energy of the desired signal and reducing the energy of the undesired signal. In this system, we introduce time reversal (TR) equalization and frequency domain equalization (FDE) to the new system and compare the performance of the two equalizations. In this paper, by MATLAB simulation, we compare the robustness to channel and the anti-interference performance of two different equalizations in the MB-IRUWB system based on MIMO.
\end{abstract}

Keywords: $M B-I R-U W B ; M I M O ; F D E ; T R$

\section{Introduction}

Ultra-wideband (UWB) is more and more interesting for its high-data rate, short range communication, slow complexity, low power spectral density and anti-multipath especially in the wireless personal area networks [1]. The frequency band used by UWB technology is up to $7.5 \mathrm{GHz}$. According to the Shannon formula, the transmission rate of the UWB system can reach Gbit/s. But, it does not reach the theoretical value in practice. In 2004, the idea of MB - IR - UWB was put forward, which can effectively improve transmission rate [2]. It can be drawn from [2-5] that, in the MB-IR-UWB, the flexibility of spectrum utilization is increased, and the system has the potential to acquire frequency diversity and multiplexing gain. [5] presents a method of subband division which is based on prolate spheroidal wave function (PSWF).

In order to further improve the performance of MB-IR-UWB, the multiple input multiple output (MIMO) technique [1] which transmits multiple signals on the space at the same time is introduced. It not only can acquire space diversity and multiplexing gain, but also can increase the channel capacity without increasing the transmission power and bandwidth. So, the MB-IR-UWB system based on MIMO which has the potential to obtain the space-time frequency diversity and multiplexing gain is presented in this paper.

However, there are inter-symbol interference (ISI), multi-stream interference (MSI) and same frequency interference (SFI) in the MB-IR-UWB system based on MIMO over multipath channel. So in order to be able to demodulate signal, an equalizer is necessary. The time reversal (TR) [6-8] can eliminate these interferences. The time reversed channel impulse response (CIR) is taken as a filter in any transmit-receive link which leads to a focus of power at the intended receiver at some particular time instant. An attractive aspect 
of TR signal processing is the fact that it makes use of multipath propagation. In addition, the rate of the system can be increased and complexity of the system is reduced. [8] proposes a TR in the UWB system based on MIMO under strong ISI, which effectively eliminate the inter-symbol interference. Meanwhile, frequency domain equalization (FDE) can also eliminate interference by converting the time domain convolution computation into frequency domain multiplication calculation. In [9], a frequency domain equalization technique is used in DS-UWB system, which wins a similar performance and low complexity to the time domain equalization. [10] proposes a TR - STBC single carrier frequency domain equalization of UWB system, using the block processing to realize high-speed data transmission.

In this article, we pay more attention to the performance of the two equalization techniques in the MB-IR-UWB based on MIMO over the multipath channel. One is TR, the FDE is another.

The rest of this paper is organized as follows. In Section 2, system model, signal model of the MB-IR-UWB system based on MIMO are described. In Section 3, the receiver of the MB-IR-UWB system based on MIMO is illustrated including equalizer and the matched filter. Two different equalizers are demonstrated. In Section 4, experimental results based on MATLAB are presented. Concluding remarks are given in Section 5.

\section{System Model, Signal Model of the MB-IR-UWB based on MIMO}

In this section, the MB-IR-UWB system based on MIMO is presented. The system model and signal model of the MB-IR-UWB system based on MIMO are given. It is that different signals are modulated by one subband pulse and transmitted in different transmit antennas. We can transmit different signals modulated by different subband pulses in one transmit antenna.

\subsection{System Model}

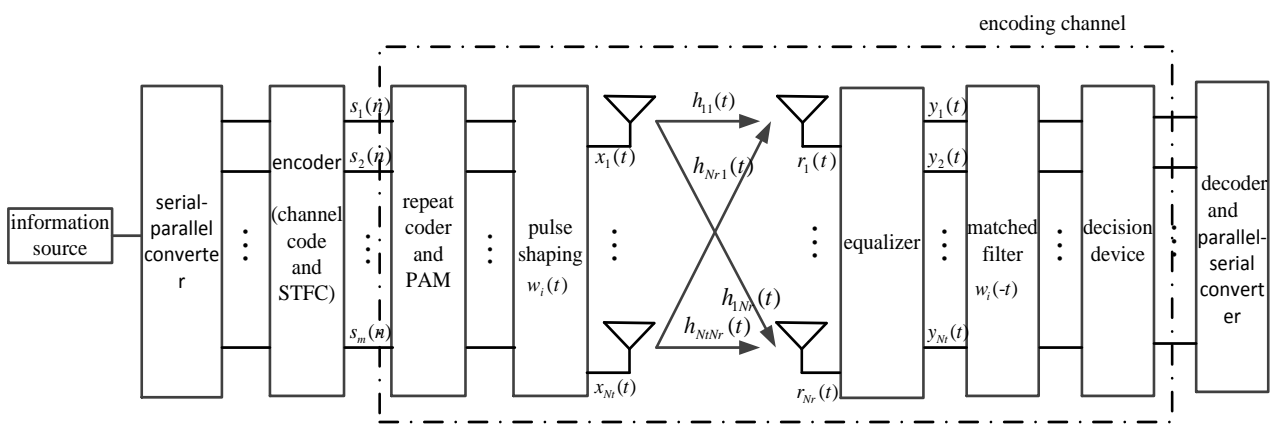

Figure 1. The Schematic Diagram for the MB-IR-UWB System based on MIMO

The schematic diagram for the MB-IR-UWB system based on MIMO is illustrated in Figure 1. Passing through a serial-parallel converter and encoder, the output signal of the information source becomes signals $s_{i}(n), i=1, \cdots, L \times N t$, where $L$ is the number of subbands. $N t$ and $N r$ denote the numbers of transmit and receive antennas. Then, $s_{i}(n)$ is fed to the repeat coder, modulator of PAM and pulse shaper $w_{i}(t), i=1, \cdots, L$, producing transmitted signals $X(t)=\left[x_{1}(t), x_{2}(t), \cdots, x_{N t}(t)\right]$. After the multipath channel $H(t)$, the receive signal $R(t)=\left[r_{1}(t), r_{2}(t), \cdots, r_{N r}(t)\right]$ is received at the receiver, where $H(t)$ is the channel matrices which is $N t \times N r$ dimensional. At the receiver, $r_{i}(t)$ is fed to the equalizer, producing signals $y_{i}(t), i=1, \cdots, N r$. Then, $y_{i}(t)$ passes though the matched filter $w_{i}(-t)$ and decision device. Finally, the signal is decoded and fed to parallel-serial converter. 
Thus, the input-output relation of the MB-IR-UWB system based on MIMO can be generally described by the following equation:

$$
\begin{aligned}
R(t) & =X(t) * H(t)+N(t) \\
H(t) & =\left[\begin{array}{ccc}
h_{11}(t) & \cdots & h_{1 N r}(t) \\
\vdots & \ddots & \vdots \\
h_{N t 1}(t) & \cdots & h_{N t N r}(t)
\end{array}\right]_{N t \times N r}
\end{aligned}
$$

where the noise is zero-mean Gaussian with PSD matrix being $N_{0}$, $N(t)=\left[n_{1}(t), n_{2}(t), \cdots, n_{N r}(t)\right]$.

\subsection{Signal Model}

On the basis of the system model mentioned above, the general transmit signal $x_{m}(t)$ at the $m$ th transmit antenna of the MB-IR-UWB system based on MIMO can be expressed in equation (3). It can be seen that different signals are modulated by different subband pulses. Meanwhile, a number of different signals are transmitted in one transmit antenna.

$$
x_{m}(t)=\sum_{l=1}^{L} \sum_{n=-\infty}^{\infty}(-1)^{s_{m l}(n)} w_{m l}\left(t-n T_{s}\right), m=1, \cdots, N t
$$

where $s_{m l}(n)$, the $n t h$ symbol after repeated coding, is modulated by the lth subband pulse at the antenna numbered with $m$. The signal adopts pulse-amplitude modulation (PAM). $T_{s}$ is the symbol duration after repeated coding. $w_{m l}(t)$ is the time domain waveform of the lth subband, transmitted at the $m t h$ antenna. The duration of $w_{m l}(t)$ is $T_{w}$. The energy carried by $w_{m l}(t)$ is normalized. The available frequency band of UWB is divided into $L$ independent subbands by the PSWF [5].

To obtain diversity and multiplexing gain of space frequency, the signal model of the proposed system is that $N t$ different signals modulated by one subband pulse are transmitted respectively from the $N t$ transmit antennas and $L \geq 2$. Then, in this article, the signal $x_{m}(t)$ transmitted at the $m t h$ antenna can be expressed as the following form.

$$
x_{m}(t)=\sum_{l=1}^{L} \sum_{n=-\infty}^{\infty}(-1)^{s_{m l}(n)} w_{l}\left(t-n T_{s}\right), m=1, \cdots, N t
$$

\section{Receiver of the MB-IR-UWB based on MIMO}

The proposed system exists MSI, SFI and ISI. In order to eliminate these interferences, we adopt equalizer at the receiver. Therefore, the receiver of the MB-IR-UWB based on MIMO mainly includes equalizer and matched filter.For the equalizer; we will give two different equalizations, TR and FDE.

\subsection{Equalizer}

3.1.1. TR: TR uses the time reversed complex conjugate of the CIR as equalizer which can focus scattered signal energy in particular time and space, no need eliminate these interferences by encoding the transmitted signal with orthogonal code.

In this system, we put TR on the receiver. Unlike the TR mentioned in [7, 8], it assumes reciprocal channel, needs to send training sequence by the receiver and estimates channel by the sender firstly, our system estimates channel and compensate the channel distortion at the receiver. In this article, we assume that the channel condition is known at the receiver. 
Let $C(t)$ denotes $N t \times N r$ dimensional TR equalizer. The mathematical expression of TR equalizer is as follows:

$$
C(t)=\left[\begin{array}{ccc}
A_{11} h_{11}(t) & \cdots & A_{N t 1} h_{N t 1}(t) \\
\vdots & \ddots & \vdots \\
A_{1 N r} h_{1 N r}(t) & \cdots & A_{N t N r} h_{N t N r}(t)
\end{array}\right\rfloor_{N r \times N t}
$$

where the entry carried by $C(t)$ is normalized, $A_{m n}=\frac{1}{\sqrt{\int_{-\infty}^{+\infty}\left|h_{m n}(t)\right|^{2} d t}}$.

Then, the output signal of TR equalizer can be expressed as equation (6).

$$
\begin{aligned}
& Y(t)=R(t) * C(t)+N(t) \\
& =X(t) * H(t) * C(t)+N(t) \\
& =X(t) *\left[\begin{array}{ccc}
r r_{11}(t) & \cdots & r r_{1 N r} \\
\vdots & \ddots & \vdots \\
r r_{N+1}(t) & \cdots & r r_{N t N \mathrm{r}}(t)
\end{array}\right]+N(t) \\
& r r_{m n}=\sum_{i=1}^{N} A_{n i} h_{m i}(t) * h_{n i}(-t)
\end{aligned}
$$

In the case ${ }^{m}=n,{ }^{r r_{m n}}$ is the superposition of autocorrelation function of the channel; in the case $m \neq n, Y(t)=\left[y_{1}(t), y_{2}(t), \cdots, y_{N t}(t)\right]$ is the superposition of cross-correlation function of the channel. The normalized autocorrelation and normalized cross-correlation function of the channel is shown in Figure 2. Table 1 gives a parameter index comparison of the autocorrelation function and cross-correlation function in different channel. According to the principle of Monte Carlo simulation, we randomly generate 500 pairs channel .The ratio is

$$
\begin{aligned}
& r_{\text {auto }}=N\left(A_{\text {auto }}>b\right) / 500 \\
& r_{\text {cross }}=N\left(A_{\text {cross }}>b\right) / 500
\end{aligned}
$$

where $A_{\text {auto }}$ is the set of the maximum sidelobe amplitude of all autocorrelation function. $A_{\text {cross }}$ is the set of the maximum amplitude of all cross-correlation function. $r_{\text {auto }}$ is ratio of maximum sidelobe amplitude of autocorrelation function larger than $b \cdot r_{\text {cross }}$ is ratio of maximum amplitude of cross-correlation function larger than $b$.

\section{Table 1. Parameter Index Comparison of the Autocorrelation Function and Cross-Correlation Function}

\begin{tabular}{|l|l|l|l|l|}
\hline Channel & $\begin{array}{l}r_{\text {auto }} \\
b=0.3\end{array}$ & $\begin{array}{l}r_{\text {cross }} \\
b=0.3\end{array}$ & $\begin{array}{l}r_{\text {auto }} \\
b=0.5\end{array}$ & $\begin{array}{l}r_{\text {cross }} \\
b=0.5\end{array}$ \\
\hline CM3 & 0.34 & 0.036 & 0.003 & 0 \\
\hline CM1 & 0.7 & 0.028 & 0.56 & 0.03 \\
\hline
\end{tabular}

It can be seen that, for $\mathrm{CM} 3$, the maximum sidelobe amplitude of all autocorrelation function almost are below 0.5. The autocorrelation function can approximate to a Dirac delta function. Almost all maximum amplitude of cross-correlation function is under 0.5; for CM1, half of the maximum sidelobe amplitude of all autocorrelation function are above 0.5 . Most of the maximum amplitude of all cross-correlation function is under 0.5. Due to TR making use of autocorrelation and cross-correlation properties of channel to compress the undesired signal, we can see that the autocorrelation function of CM3 is closer to an impulse function than CM1. The cross-correlation function of CM3 is closer to zero than CM1. Therefore, the performance of TR in CM3 outperforms the performance of TR in CM1. 
In view of the above illustration of channel autocorrelation and cross-correlation function, we can ignore the channel cross-correlation function and the system is simplified to the following formula

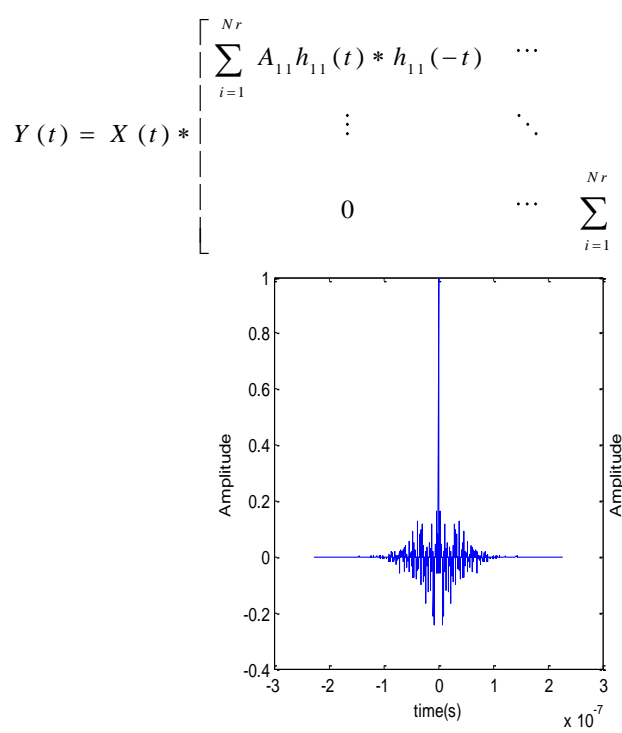

(a)
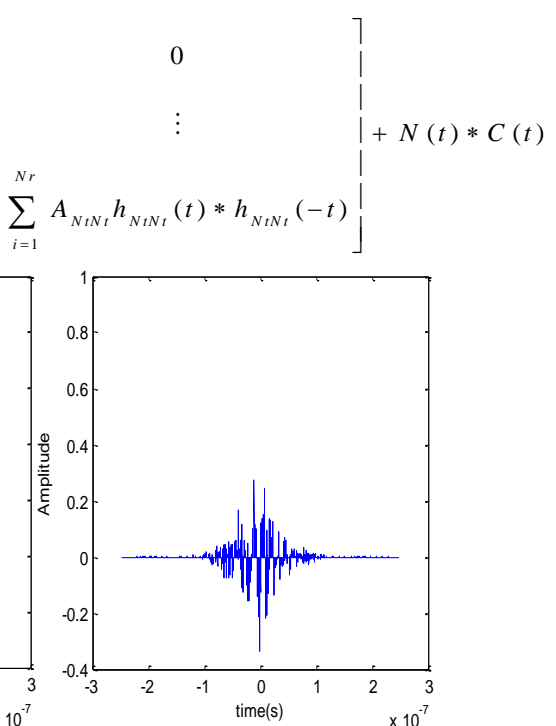

(b)

Figure 2. The Auto-correlation Function (a) and the Cross-correlation Function (b) of Channel

3.1.2. FDE: The schematic diagram for FDE in the MB-IR-UWB system based on MIMO is illustrated in Figure 3.

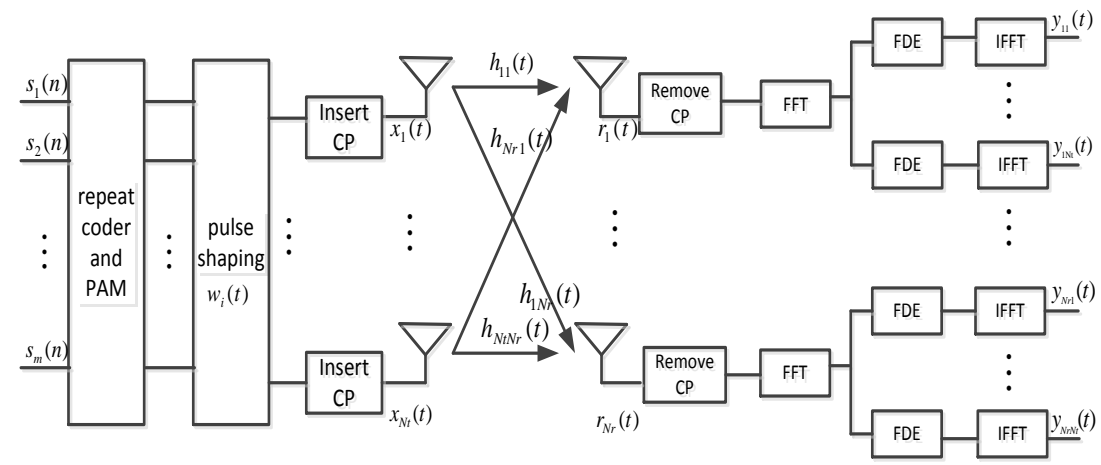

Figure 3. The Schematic Diagram for FDE of the MB-IR-UWB System based on MIMO

From Figure 3, we can see that, by inserting cyclic prefix, time domain convolution operation can be translated into product operation of frequency domain. The cyclic prefix $u w$ is inserted to signal transmitted in each antenna, and the length of $u w$ must larger than the length of channel. After inserting cyclic prefix, we get transmit signal $x_{i}(t), i \in\{1,2, \cdots, N t\}$. Passing though the multipath channel, the signal received in $k t h$ antenna is shown by (11) in time domain.

$$
r_{k}(t)=\sum_{i=1}^{N t} x_{i}(t) * h_{i k}(t)+n_{k}(t)
$$


where signals transmitted by different antennas are independent, i.e. $E\left[x_{k} x_{l}^{T}\right]=0$ for $k \neq l$. In addition, all transmitted signals and noise are statistically independent, $E\left[x_{k} n_{l}^{T}\right]=0$ for $\forall k \in\{1,2, \cdots, N t\}, l \in\{1,2, \cdots, N r\}$.

At the receiver, the step of the frequency domain equalization is as follows. The first to do is removing cyclic prefix. Then the signal is processed by the FFT, converting the receive signal to the frequency domain which is shown as follows.

$$
R_{k}=\sum_{i=1}^{N t} H_{i k} X_{i}+N_{k}
$$

where $H_{i k}$ is the frequency domain coefficient of channel from ith transmitter to $k t h$ receiver. $R_{k}$ and $X_{i}$ are the frequency domain coefficient of $k t h$ received signal $r_{k}(t)$ and ith transmitted signal $x_{i}(t)$, respectively. $N_{k}$ is the frequency domain coefficient of $k t h$ noise $n_{k}(t)$.

To demodulate the signal transmitted by the $j t h$ antenna, the received signal $R_{k}$ is divided into two parts, one is the signal subspace, one is the interference subspace. Then, the received signal $R_{k}$ can be written as follows.

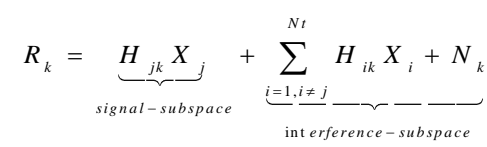

After FFT, the received signal $R_{k}$ is processed by equalizer $U_{j k}$, producing estimated signal $Y_{j k}$ for signal transmitted by $j$ th antenna.

$$
Y_{j k}=U_{j k}^{H} R_{k}=U_{j k}^{H} H_{j k} X_{j}+U_{j k}^{H} \sum_{i=1, i \neq j}^{N t} H_{i k} X_{i}+U_{j k} N_{k}
$$

In order to find qualified equalizer $U_{j k}$, we use the minimum mean square error (MMSE) as the cost function, and find $U_{j k}$ to minimize the mean square error. So, this process can be expressed as follows. The mean square error (MSE) can be defined as (15).

$$
\varepsilon_{j k}=E\left[\left\|Y_{j k}-X_{i}\right\|^{2}\right]
$$

Equalizer $U_{j k}$ is optimized by minimizing the mean square error subject to the transmit power constrain $P$.

$$
U_{j k}^{M M S E}=\underset{U_{j k}}{\arg \min \varepsilon_{j k}} \text { subject to }\left\|X_{j}\right\|_{2}^{2} \leq P
$$

This function can be solved by a Lagrange function (17).

$$
L=\varepsilon_{j k}+\lambda_{k}\left[\operatorname{trace}\left(X_{j} X_{j}^{H}\right)-P\right]
$$

where $\lambda_{k}$ denotes Lagrange multiplier. The optimal equation can be solved by the following function.

$$
\nabla L=0
$$

By the above equations, the optimal equalizer ${ }^{U}{ }_{j k}$ can be solved by equation $\nabla_{U_{j k}} L=0$, and we can get it as (19) [11].

$$
U_{j k}^{M M S E}=\left(\sum_{i=1}^{N t} H_{i k} H_{i k}^{H}+\sigma^{2} I\right)^{-1} H_{j k}
$$

Then, the interference subspace is compressed, and $\left(U_{j k}^{M M S E}\right)^{H} H_{j k}$ tends to a constant. The output signal of equalizer is expressed as (20).

$$
Y_{j k}=\left(U_{j k}^{M M S E}\right)^{H} R_{k} \approx\left(U_{j k}^{M M S E}\right)^{H} H_{j k} X_{j}+\hat{N}_{k}
$$

where $\hat{N}_{k}$ denotes the nose after equalizer. 
The $Y_{j k}$ passes through IFFT, producing the time domain signal $y_{j k}(t)$.Finally, for all $k \in\{1,2, \cdots, N t\}$, the received signal of is expressed as (21).

$$
\begin{gathered}
Y(t)=\left[y_{1}(t), y_{2}(t), \cdots, y_{N t}(t)\right] \\
y_{j}(t)=\sum_{k=1}^{N r} y_{j k}(t)=\sum_{k=1}^{N r} g_{j k}(t) * h_{j k}(t) * x_{j}(t)
\end{gathered}
$$

where $g_{j k}(t)$ is the time domain equalizer of FDE. $g_{j k}(t)=\operatorname{IFFT}\left[\left(U_{j k}^{M M S E}\right)^{H}\right]$.

\subsection{Matched Filter}

The received signal passes the equalizer and yields the signal $Y(t)=\left[y_{1}(t), y_{2}(t), \cdots, y_{N_{t}}(t)\right]$. Where $y_{i}(t)$ denotes a signal corresponding to the signal transmitted from the ith antenna .It's duration is a bit duration. $y_{i}(t)$ is given by the equation (23).

$$
\begin{aligned}
& y_{i}(t)=x_{i}(t) * \sum_{l=1}^{N r} h_{i l}(t) * g_{i l}(t)+\sum_{l=1}^{N r} n_{i}(t) * g_{i l}(t) \\
& =\sum_{j=1}^{L} a_{i j} * w_{i j}(t) * \sum_{l=1}^{N r} h_{i l}(t) * g_{i l}(t)+\sum_{l=1}^{N r} n_{i}(t) * g_{i l}(t)
\end{aligned}
$$

where $a_{i j}=(-1)^{s_{i j}}$ is the PAM symbol, transmitted from the $i t h$ antenna and conveyed by the $j$ th subband pulse $w_{i j}(t) \cdot g_{i l}(t)$ denotes the equalizer. For TR, $g_{i l}(t)=h_{i l}(-t)$; for FDE, $g_{i l}(t)=\operatorname{IFF}\left[\left(U_{i l}^{M M S E}\right)^{H}\right] \cdot h_{e}$, a equivalent channel which is shown in (24), tends to a impulse function.

$$
h_{e}=\sum_{i=1}^{N r} h_{i l}(t) * g_{i l}(t)
$$

Due to the subband pluses are mutually independent, the signal after matched filter $w_{i n}(-t)$ is as follows:

$$
\begin{aligned}
& y_{i}(t) * w_{i n}(-t) \\
& =\sum_{j=1}^{L} a_{i j} w_{i j}(t) * \sum_{l=1}^{N r} h_{i l}(t) * g_{i l}(t) * w_{i n}(-t)+\sum_{l=1}^{N r} n_{i}(t) * g_{i l}(t) * w_{i n}(-t) \\
& =a_{i n} w_{i n}(t) * w_{i n}(-t) * \sum_{l=1}^{N r} h_{i l}(t) * g_{i l}(t)+\sum_{l=1}^{N r} n_{i}(t) * g_{i l}(t) * w_{i n}(-t)
\end{aligned}
$$

When $t=0$, if $y_{i}(t) * w_{i n}(-t) \geq 0$, the symbol transmitted at the $i t h$ antenna is sentenced to 0 , otherwise the symbol is sentenced to 1 .

Note that, defining the signal-to-noise ratio per antenna as $E_{b} / N_{0}, E_{b}$ is the mean bit energy per transmitted antenna conveyed by one subband after multipath channel. The channel is assumed to be a static channel during the frame period. So, we can compare the BER performance of the MB-IR-UWB based on MIMO with the SB (signal band) SISO system, ensuring that the signal-to-noise ratio is the same after every matched filter in the encoding channel.

\section{Simulation Results}

In this section, we will give BER performance of different equalizer in the MB-IRUWB based on MIMO so as to compare the performance of the two equalizer in the system.

Table 2 gives simulation parameters of the MB-IR-UWB based on MIMO with different equalizer. A string of guard symbol is added after the information symbol to make the symbol duration $T_{s}$ greater than the channel delay spread $T_{s}$ to avoid ISI or make the symbol duration $T_{s}$ less than the channel delay spread $T_{s}$ to make the system exist 
ISI , where $T_{s}=n h T_{w}$ and we add guard symbol with duration $(n h-1) T_{w}$ after the information symbol. The Monte Carlo method is applied to evaluate the performance of the MB-IRUWB based on MIMO with different equalizer .

Table 2. Simulation Parameters

\begin{tabular}{|c|c|c|}
\hline Parameter & Symbol & Value \\
\hline Frequency bounds & $B$ & $3.1 \mathrm{GHz}-10.6 \mathrm{GHz}$ \\
\hline Number subband & $L$ & 15 \\
\hline Bandwidth of subband & $w$ & $500 \mathrm{MHz}$ \\
\hline Pulse type & - & PSWF \\
\hline Pulse duration & $T_{w}$ & $3 \mathrm{~ns}$ \\
\hline $\begin{array}{lll}\text { Symbol repetition } & \text { period } \\
\text { without ISI } & & \end{array}$ & $T_{s}$ & $21 \mathrm{~ns}$ \\
\hline $\begin{array}{l}\text { Duration of guard symbol } \\
\text { without ISI }\end{array}$ & $(n h-1) T_{w}$ & $18 \mathrm{~ns}$ \\
\hline $\begin{array}{l}\text { Symbol repetition period with } \\
\text { ISI }\end{array}$ & $T_{s}$ & $9 \mathrm{~ns}$ \\
\hline $\begin{array}{l}\text { Duration of guard symbol with } \\
\text { ISI }\end{array}$ & $(n h-1) T_{w}$ & $6 \mathrm{~ns}$ \\
\hline Modulation & - & PAM \\
\hline Channel model & - & CM3,CM1 \\
\hline Channel delay spread for CM1 & $T_{g}$ & $6 \mathrm{~ns}$ \\
\hline Channel delay spread for CM3 & $T_{g}$ & $18 \mathrm{~ns}$ \\
\hline
\end{tabular}

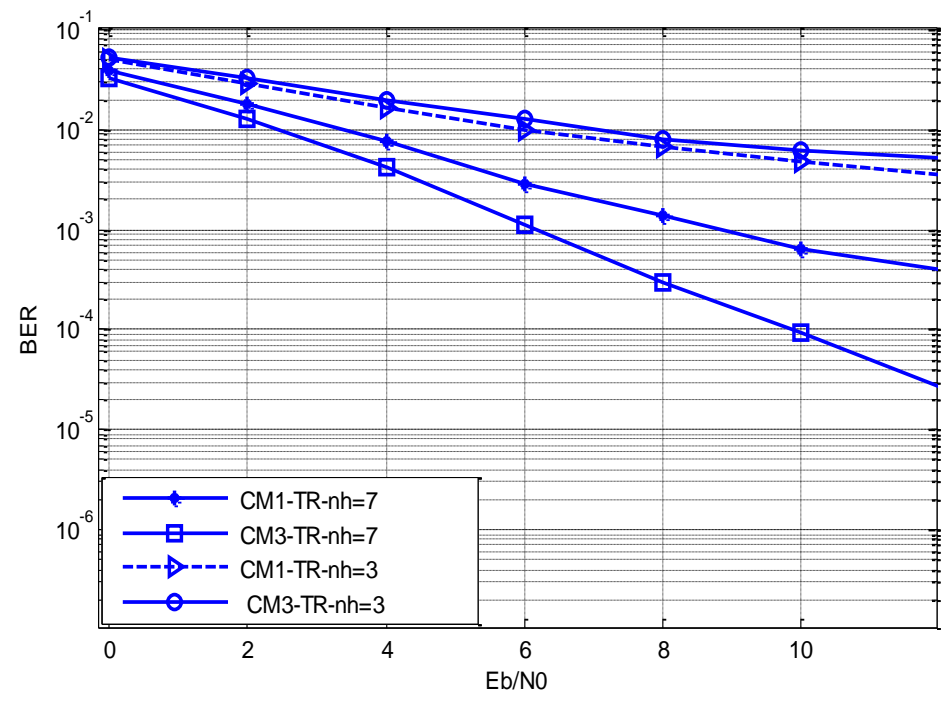

Figure 4. The BER Performance of the MB-IR-UWB based on MIMO with TR

The BER performance of the MB-IR-UWB based on MIMO with TR is demonstrated in Figure 4. As is shown in Table 1, the TR performance is related to the channel correlation. From Figure 4, it can be seen that the BER performance of TR is considerably increased with the increase of the symbol duration $T_{s}$, which is a result of the increasing of the symbol duration makes system having a increasing ability to avoid ISI. The BER performance over CM3 outperforms the BER performance over CM1 3dB at the BER is $10^{-3}$ when the system has no ISI, i.e., $n h=7$; the BER performance over CM3 and the BER performance over CM1 are similar when $n h=3$. When $n h=3$, system over CM3 has 
ISI, while system over CM1 has no ISI. The simulation results are because of the correlation of CM3 is better than CM1, which lead to the BER performance over CM3 better than the BER performance over CM1, even in the case of the comparison about system with ISI over CM3 and system without ISI over CM1. It can be shown that the BER performance of the MB-IR-UWB based on MIMO with TR is different with the different channel and ISI. And CM3 is more suitable for the TR than CM1.

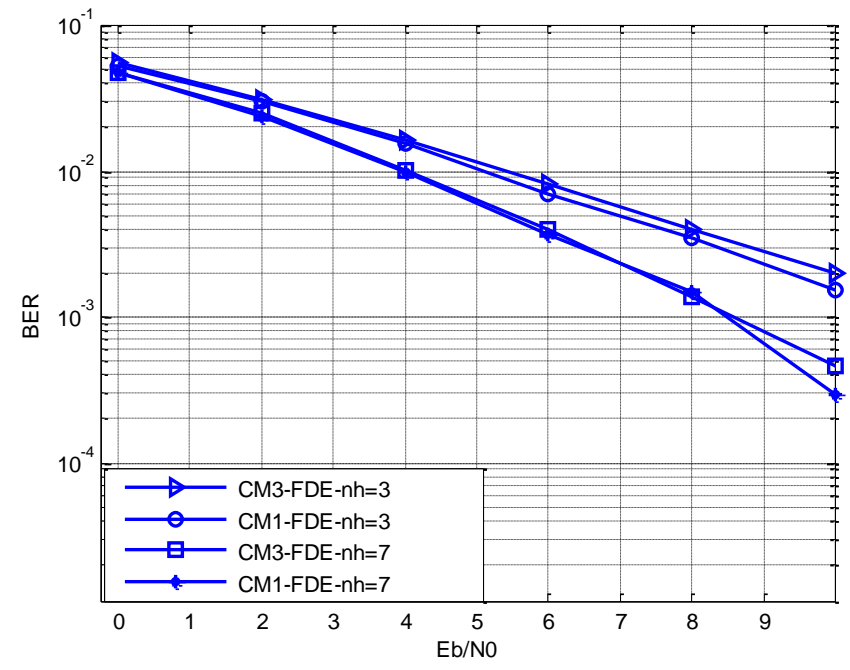

Figure 5. The BER Performance of the MB-IR-UWB based on MIMO with FDE

Figure.5 gives the BER performance of the MB-IR-UWB based on MIMO with FDE. It can be seen that the BER performance of the MB-IR-UWB based on MIMO with FDE is almost the same for different channel; the BER performance can be considerably increased with the increase of the symbol duration $T_{s}$. The result displays that the FDE has a better robustness for different UWB channel than the TR in MB-IR-UWB based on MIMO. And the performance of FDE has a relationship with symbol duration $T_{s}$.

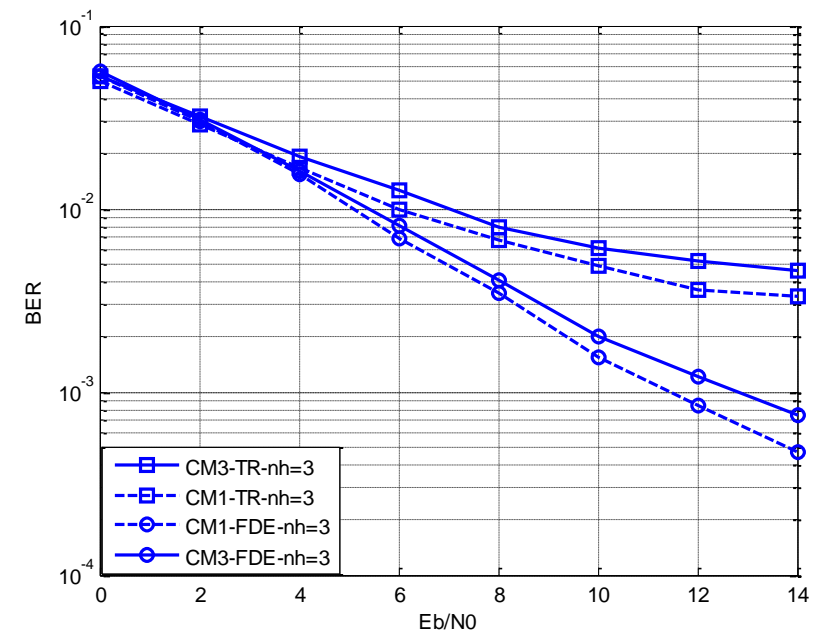

Figure 6. The BER performance of the MB-IR-UWB based on MIMO with Different Equlizer in the Case of $n h=3$ 
Figure 6 gives the BER performance of the MB-IR-UWB based on MIMO with different equlizer in the case of $n h=3$. When $n h=3$, system over CM3 has ISI, while system over CM1 has no ISI. From the Figure 6, it can be observed that the BER performance of FDE outperforms the TR even in the case of the comparison about system with ISI over CM3 and system without ISI over CM1.We can see that the FDE has better ability to resist interference than TR with ISI.

The BER performance of the MB-IR-UWB based on MIMO with different equalizer without ISI, i.e., $n h=7$ is illustrated in Figure 7. From Figure 7, we can see that for the $\mathrm{CM} 3$, the BER performance of TR outperforms FDE; for the CM1, the BER performance of TR outperforms FDE in low SNR, while the BER performance of TR is poorer than FDE in high SNR. This means that when the correlation of channel is good enough, the antiinterference of TR is better than FDE in the case of system without ISI. As the correlation of channel increasingly unsatisfactory, the anti-interference of TR can decrease to the extent that the performance is poorer than FDE in the case of system without ISI.

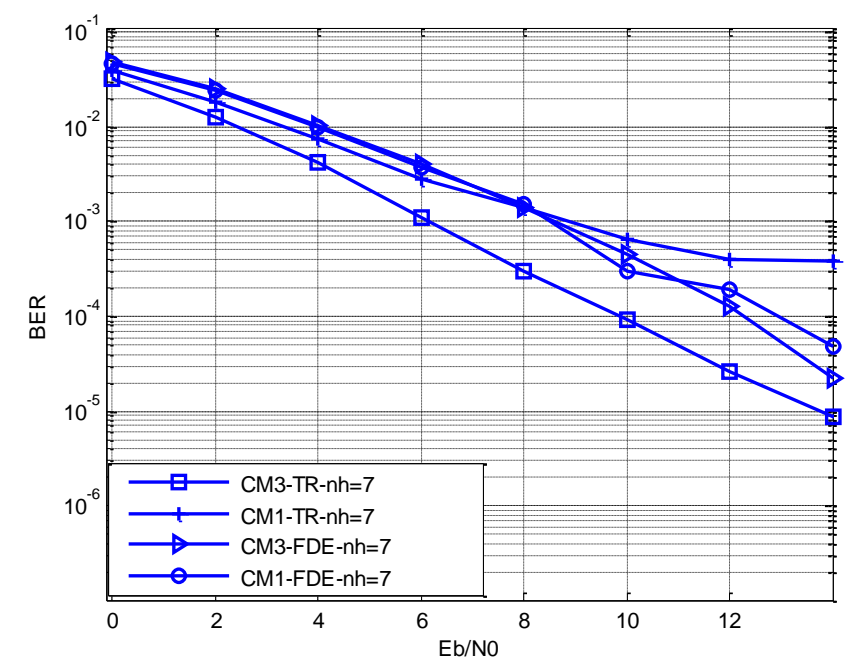

Figure 7. Tthe BER Performance of the MB-IR-UWB based on MIMO with Different Equlizer without ISI

\section{Conclusion}

This paper compares the performance of two different equlizer in a new system, the MBIR-UWB system based on MIMO. One of the two equlizer is TR equlizer, and the other one is FDE. The system model and signal model of MB-IR-UWB system based on MIMO are given. We also show the theory of the two equlizer to eliminate MSI, SFI and ISI over the multipath channel proposed by IEEE 802.15.3a. By compare BER performance in different conditions, we can draw the following conclusions. On the one hand, as long as the channel correlation is good enough, TR equalizer's anti-interference performance will be better than the FDE for the system without ISI; for the system with ISI, the performance of FDE outperforms the TR. So, it can be shown that FDE has a better anti-ISI performance than the TR;TR equalizer's performance of anti-MSI,anti-SFI is better than FDE as long as the channel correlation is good enough. On the other hand, the channel robustness of FDE outperforms the TR.

\section{Acknowledgement}

Many thanks to my supervisor Prof.Qi Lin and other fellow workers for the guidance, encouragement and support during the period of this work. This research was supported by 
the National Science Nature Foundation of China under Grant No 61201237 and No 61401115. This research was also supported by the Defense Industrial Technology Development Program under Grant No J083814001.

\section{References}

[1] T. Kaiser, F. Zheng, E. Dimitrov, “An overview of ultra-wide-band systems with MIMO”, vol. 2, no. 97, (2009).

[2] S. Paquelet, L. M. Aubert, B. Uguen, "An impulse radio asynchronous transceiver for high data rates", (2004); Rennes, France.

[3] M. Mittelbach, R. Moorfeld and A. Finger, "Performance of a multiband impulse radio UWB architecture", (2006); New York, USA

[4] A. Hasan, A. Anwar, H. Mahmood, "On the performance of multiuser multiband DSUWB system for IEEE 802.15. 3a channel with hybrid PIC rake receiver",(2011); Abbottabad, Pakistan

[5] L. Chen and Z. Dou, "Performance Analysis of Multiband Impulse Radio UWB Communication System Based on PSWF", vol. 5, no. 161, (2013).

[6] T. Strohmer, M. Emami, J. Hansen, G. Papanicolau, and A.J. Paulraj, "Application of timereversal with MMSE equalizer to UWB communications", (2004).

[7] C. Zhou, N. Guo and R. C. Qiu, "Time-reversed ultra-wideband (UWB) multiple input multiple output (MIMO) based on measured spatial channels", vol. 6, no. 58, (2009).

[8] W. Taotao, Lv Tiejun, "Space-Time Pre-equalization for Time Reversal MIMO UWB System in Strong ISI", (2011).

[9] P. Kaligineedi, V. K. Bhargava, "WLC18-3: Frequency-Domain Equalization Techniques for DS-UWB Systems", (2006).

[10] W. Dan, J. Lingge and H. Chen, "A MIMO Transceiver Scheme Using TR-STBC for SingleCarrier UWB Communications with Frequency Domain Equalization”, (2007).

[11] C. Le, E. Dimitrov, S. Moghaddamnia and T. Kaiser, "Performance investigation of MMSEbased interference alignment for multiuser MIMO UWB”, (2011). 
International Journal of Multimedia and Ubiquitous Engineering

Vol. 10, No. 3 (2015) 